\title{
ULTRA-VIOLET REFLECTING POWER OF SOME METALS AND SULPHIDES
}

\author{
By W. W. Coblentz and C. W. Hughes
}

ABSTRACT

This paper gives data on the ultra-violet reflecting power of the sulphides of metals (lead, molybdenum, iron, and antimony) having a high metallic luster. The materials examined were the natural minerals-galena, molybdenite, pyrites, and stibnite.

It is shown that, in contrast with the metals which have a low reflecting power in the ultra-violet and in the visible spectrum, followed by a high reflecting power in the infra-red, the sulphides of these metals have a high selective reflection in the ultra-violet, followed by lower and more uniform reflection in the infra-red spectrum. Data are given also on the reflecting power of graphite, duralumin, and magnalium.

\section{CONTENTS}

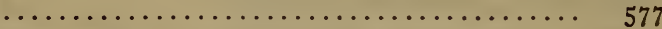

II. Apparatus and procedure.................................. $57^{8}$

III. Reflectivity measurements................................ $5^{80}$

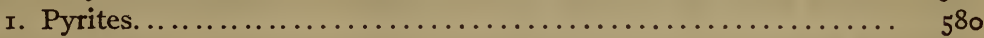

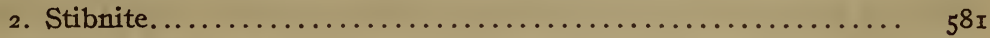

3. Molybdenite...................................... $5^{8 \mathrm{I}}$

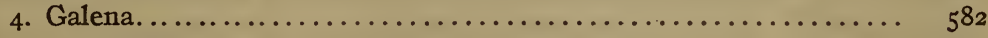

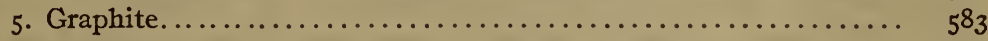

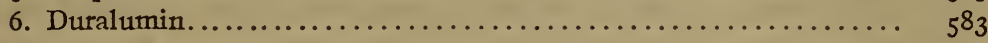

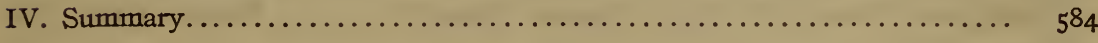

\section{INTRODUCTION}

The investigations of Hagen and Rubens, ${ }^{1}$ of Hulburt, ${ }^{2}$ and of others show that most of the metals have a low reflectivity in the ultra-violet, which increases in value with increase in wave length. On the other hand, no data are at hand concerning the ultraviolet reflecting power of the sulphides of metals (for example, antimony, iron, molybdenum, etc.) which have a high metallic luster and which seem to have spectral reflective properties that are just the reverse of the metals, viz, a high reflectivity in the visible spectrum ${ }^{3}$ followed by a low and spectrally uniform reflecting power in the infra-red. ${ }^{4}$

\footnotetext{
1 Hagen and Rubens, Ann. der Phys., 8, p. r; 1902.

${ }^{2}$ Hulburt, Astrophys. Jour., 42, p. 205; r9r5.

3 Coblentz and Kahler, B.S. Sci. Papers (No. 338), 15, p. I21; 19r9; Tyndall, Phys. Rev. (2), 21, p. I62; ז923.

Coblentz, Publ. No. 65, Carnegie Inst. of Wash.; I906; Crandall, Phys. Rev. (2), 2, p. 343; I9r3.

$4890^{\circ}-24$ 
The high selective reflection of galena, stibnite, and molybdenite in the violet is easily observed by holding two cleaved pieces of the mineral so as to form a V-shaped opening which permits multiple reflection of daylight.

There are important applications in which use could be made of mirrors having a high reflecting power in the ultra-violet and a low reflection of visible and infra-red rays. A specific example, which prompted this investigation, was an inquiry for a suitable reflector for a laryngoscope mirror to be used in the treatment of the throat by means of sunlight, which contains intense visible and infra-red radiation having no marked therapeutic properties, but capable of causing burns. Hence, in this particular application it is desirable to eliminate the infra-red rays.

\section{APPARATUS AND PROCEDURE}

The arrangement of the apparatus for determining the reflecting power of the substance under investigation was essentially the same as in previous work. ${ }^{5}$

The reflecting properties of the unknown sample were compared with that of metals whose spectral reflectivities are known. For this purpose the two mirrors were mounted side by side upon a suitable sliding mechanism which could be moved in front of the entrance slit of the spectroradiometer. By means of a quartż lens light from a quartz mercury lamp was concentrated upon one of these mirrors and from thence brought to a focus upon the entrance slit.

The quartz spectropyrheliometer previously described ${ }^{6}$ with a vacuum thermopile was adapted to this work. For this purpose the focus of the entrance slit was kept constant and the exit slit (the thermopile) was focused upon the different spectral lines. This was an easy task, since the thermopile slit was provided with a fluorescent screen (anthracene) which rendered the ultra-violet lines visible to the eye. ${ }^{7}$

With the high light-gathering power of this apparatus and the sensitive vacuum thermopile, observations at $250 \mathrm{~m} \mu$ could be made as accurately (galvanometer deflection of 8 to $10 \mathrm{~cm}$ ) as in the more intense part of the spectrum. However, high accuracy is not the only thing to be considered. All the materials examined (cobalt, steel, nickel, magnalium, etc.) were found to be affected

8 Coblentz and Emerson, B. S. Sci. Papers (No. 308), 14, p. 307; x9r7.

6 Coblentz and Kahler, B. S. Sci. Papers (No. 378), 16, p. 233; 1920.

i Coblentz, Jour. Opt. Soc. Am., 7, p. 45x; x923. 
by ultra-violet radiation. This was easily ascertained by blowing the breath upon the mirror after it had been exposed for awhile to the concentrated radiation from the quartz lamp. While this deterioration (which is probably caused by oxidation) was not perceptible visually, it had some effect of lowering the reflecting power in the short wave lengths at 250 to $300 \mathrm{~m} \mu$. The quartz concentrating lens was, therefore, kept covered with a thick piece of plate glass while making the preliminary adjusments, and the reflectivity of the short wave lengths was determined first in order to avoid the effect of this deterioration. Before examination the samples were polished on chamois skin charged with rouge, which readily removed the effects of the ultra-violet radiation. ${ }^{8}$

A slight difference in polish has an appreciable effect upon the absolute values of reflectivity. For example, a comparison was made of the reflecting power of two samples of steel which differed perceptibly in polish, and it was found that the more highly polished sample had a uniformly higher reflecting power, amounting to about 4 per cent, throughout the ultra-violet spectrum.

On the other hand, two selenium mirrors which had been cast on glass and appeared the same color to the eye, differed markedly in their reflectivity in the extreme ultra-violet spectrum. Mirrors which are deposited cathodically upon glass seem to be easily affected by surface films which may not be perceptible to the eye. Mirrors of the massive metal were, therefore, used as standards. The reflectivity of some of the mirrors used as standards of comparison had previously been determined by Hulburt, using a direct measurement of the absolute value.

The absolute values of reflectivity plotted in the illustrations were obtained by multiplying the relative (to the standard) values by the absolute values of the standard mirrors as observed by Hagen and Rubens and by Hulburt (loc. cit.). Many of these metals have a minimum reflectivity in the region of 250 to $300 \mathrm{~m} \mu$, which seems to be slightly different (for nickel) as observed by different observers. Cobalt and tantalum have a smooth reflectivity curve in this region of the spectrum, hence they are the best adapted for determining the shape of the reflectivity curve of the unknown sample.

\footnotetext{
8 In previous investigations of the ultra-violet reflecting power of metals no mention is made of the corrosion of polished surfaces by the action of ultra-violet radiation, which seems to accelerate atmospheric corrosion. These results seem to be novel and are being investigated by the division of metallurgy with the hope of developing a new method for testing the liability of metals to corrosion, etc., to replace the chemical tests heretofore employed.
} 
The surfaces of the sulphide crystals were not entirely free from imperfections, hence it is impossible to give their exact absolute values of reflectivity, which might be 5 to ro per cent higher.

It seems of most interest at present to determine the reflectivity in the ultra-violet relative to that of the visible and the infra-red spectrum. By comparing the unknown sample (for example, pyrites) with several standard materials ( $\mathrm{Co}, \mathrm{Ni}, \mathrm{Ta}, \mathrm{Si}$ ) an average reflectivity curve is obtained which is considered more reliable than that resulting from a comparison with a single standard.

Just why so many metals have a minimum reflecting power in the region of $250 \mathrm{~m} \mu$ has not yet been determined. This is a question worthy of investigation.

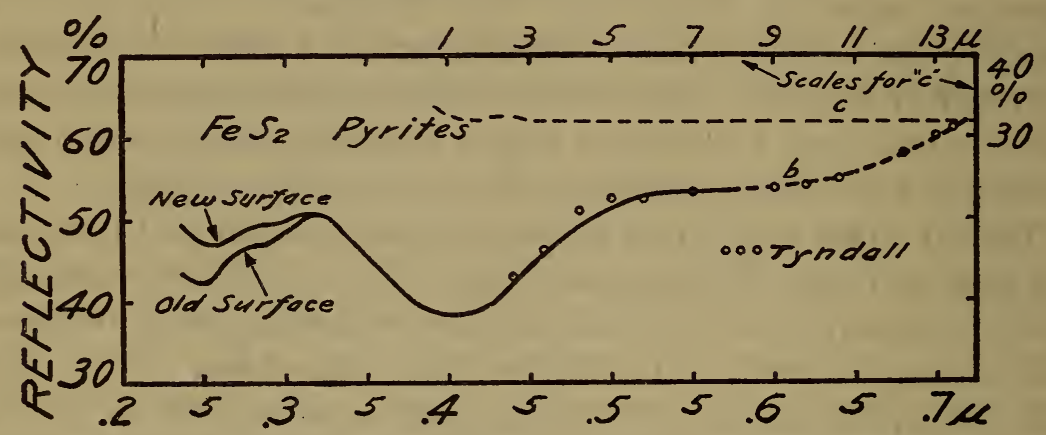

Fig. I.-Reflecting power of pyrites in various parts of the spectrum

\section{REFLECTIVITY MEASUREMENTS}

\section{PYRITES $\left(\mathrm{FeS}_{2}\right)$}

I. The material examined was kindly loaned by Dr. F. E. Wright, of the geophysical laboratory. One large sample had a crystal face 5 by $5 \mathrm{~cm}$. Two smaller crystals had faces 2 by 3 cm. In all samples the faces were perfectly flat and highly polished, but with several strias extending lengthwise across the surface. In this material the above-mentioned deterioration of the surface by exposure to ultra-violet radiation did not occur to a marked extent. Nevertheless, as shown in Figure I, the effect upon the reflectivity of an old and a newly polished surface is quite perceptible in the region of 240 to $320 \mathrm{~m} \mu$.

The reflectivity curve of pyrites given in Figure $\mathrm{I}$ is the average of the comparisons with cobalt, tantalum, tungsten, and silicon, which mirrors had been examined by Hulburt (loc. cit.). The data in the violet end of the visible spectrum agree remarkably well with the spectrophotometric measurements made by Tyndall (loc. cit.), as shown in curve $b$ of Figure $\mathrm{I}$. 
In a previous investigation ${ }^{9}$ it was shown that the reflecting power of pyrites is uniform throughout the infra-red spectrum (to $12 \mu$ ) and is somewhat lower than in the visible spectrum, as depicted in curve $c$ of Figure $\mathrm{I}$.

\section{STIBNITE $\left(\mathrm{Sb}_{2} \mathrm{~S}_{3}\right)$}

2. The material came from Japan. The sample examined was an unusually brilliant cleaved surface which was optically flat over an area of about 15 by $25 \mathrm{~mm}$.

The reflectivity in the ultra-violet is illustrated in curve $a$ of Figure 2. The data at 240 to $365 \mathrm{~m} \mu$ were obtained by comparison with a cobalt mirror which has a smooth reflectivity curve in this spectral region. From this it is concluded that the indentation at $250 \mathrm{~m} \mu$, which, as already stated, is found in many

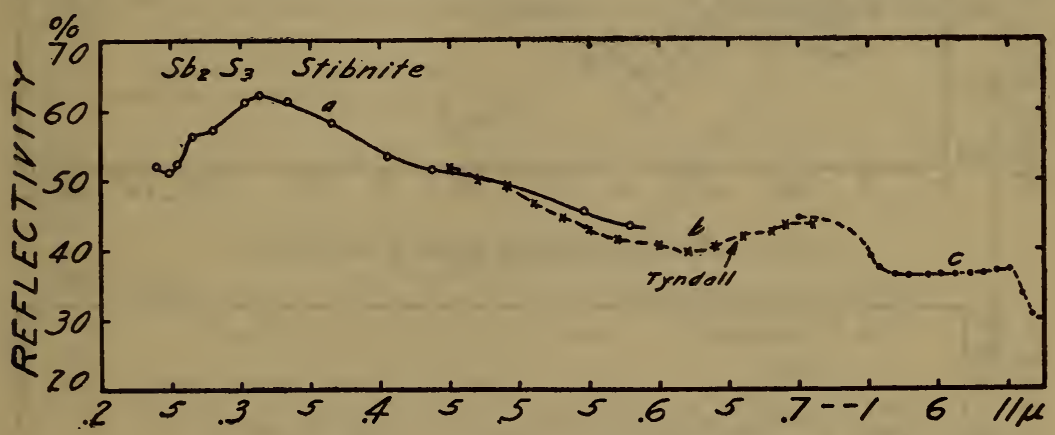

FIG. 2.-Spectral reflectivity of stibnite

metals, is a property of this material. That part of curve $a$ lying between 365 and $579 \mathrm{~m} \mu$ is based mainly upon a comparison with a mirror of nickel sawed from a rod $32 \mathrm{~mm}$ in diameter. ${ }^{10}$ The data are in good agreement with the spectrophotometric measurements, curve $b$, obtained by Tyndall. ${ }^{11}$

The infra-red reflectivity ${ }^{12}$ of stibnite, extending from I to II $\mu$ is illustrated in curve $c$, Figure 2. From this it appears that stibnite has a reflecting power of about 60 per cent at $0.3 \mu$ in the ultra-violet and about 40 per cent in the visible spectrum. In the infra-red the reflectivity is uniform and amounts to about 35 per cent.

\section{MOLYBDENITE $\left(\mathrm{MoS}_{2}\right)$}

3. The material examined came from Renfrew Co., Canada. Thin cleaved laminas ( 2 by $3 \mathrm{~cm}$ in area) were pressed out smooth

- Coblentz, Publ. No. 65, Carnegie Inst. of Wash., p. $91 ; 1906$.

10 Kindly supplied by Dr. P. D. Merica, The International Nickel Co., Bayonne, N. J.

11 Tyndall, Phys. Rev. (2), 21, p. I66; I923.

12 Coblentz, Publ. No. 65, Carnegie Inst. of Wash., p. 91 ; 1906. 
upon flat plates of glass. The surfaces were highly polished, but contained a few strias and rough streaks which remained from crimping, caused while splitting off the lamina. While these rough, unpolished streaks do not affect the relative values of spectral reflection, they reduce the absolute values by a small amount.

The ultra-violet reflecting power of two samples of molybdenite was investigated. As illustrated in Figure 3, the rapid rise in reflecting power, previously observed ${ }^{13}$ in the visible spectrum, terminates in a maximum at about $450 \mathrm{~m} \mu$. A low reflectivity occurs at $340 \mathrm{~m} \mu$, and a second maximum at about $250 \mathrm{~m} \mu$.

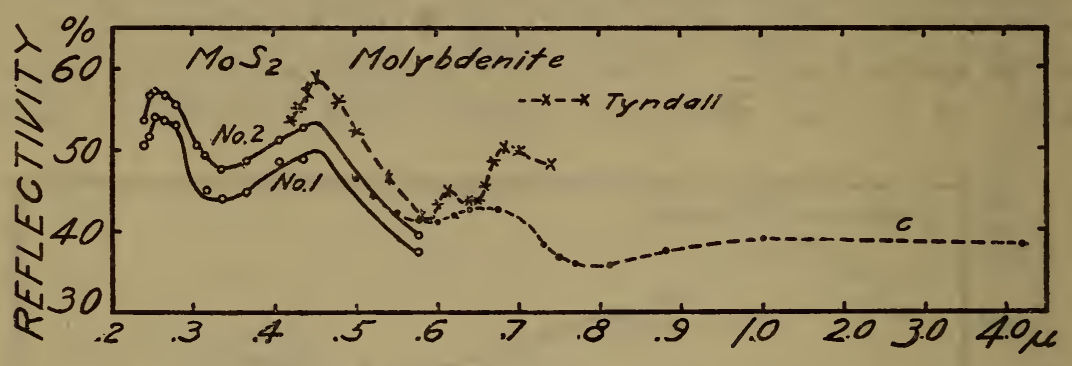

FrG. 3.-Spectral reflecting power of molybdenite

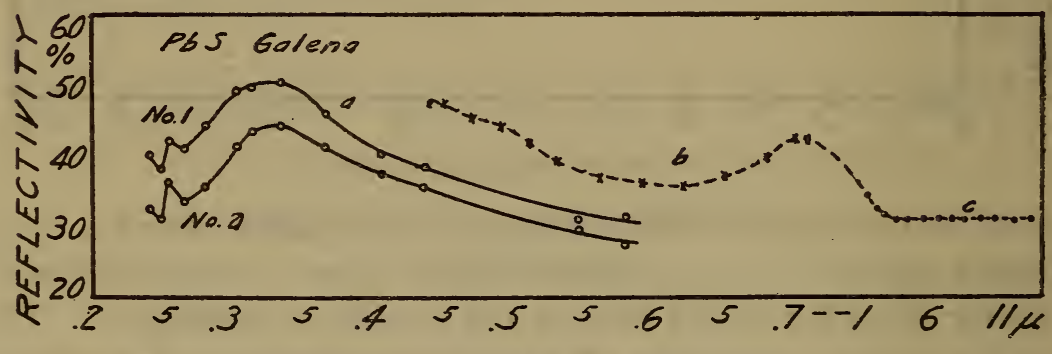

FIG. 4.-Spectral reflecting power of galena

The general outline of the reflectivity curve at $450 \mathrm{~m} \mu$ agrees well with Tyndall's (loc. cit.) spectrophotometric measurements.

The reflecting power in the infra-red curve $c$, Figure 3 , is much lower, and it is uniform, as observed in previous ${ }^{14}$ investigations.

\section{GALENA (Pb S)}

It is difficult to obtain flat cleaved surfaces on galena crystals. The two samples examined were fresh cleaved surfaces, which were quite flat over an area of about ro by ro $\mathrm{mm}$.

The ultra-violet reflectivity measurements are illustrated in Figure 4 , in which curve $b$ shows Tyndall's (loc. cit.) spectrophotometric observations.

${ }^{13}$ Coblentz, and Kahler, B. S. Sci. Papers (No. 338), 15, p. r2x; r9r9.

11 B. S. Sci. Papers (No. 338); 1919; and Carnegie Publ. No. 65 :1906. 
In the infra-red spectrum, curve $c$, the reflecting power is lower and uniform (to I2 $\mu$ ), which is as far as the observations have yet been made.

From these data it appears that galena has a wide maximum of reflectivity at about $325 \mathrm{~m} \mu$ in the ultra-violet and another, smaller, maximum at about $700 \mathrm{~m} \mu$, followed by a fairly uniform reflectivity in the infra-red.

\section{GRAPHITE (C)}

It was previously found that graphitized carbon has a high reflecting power, especially for infra-red rays. ${ }^{15}$ There is but little difference in the reflectivity of homogeneous surfaces of artificial graphite and the natural (Siberian) mineral. The infrared observations, previously observed at I to I I $\mu$, are depicted

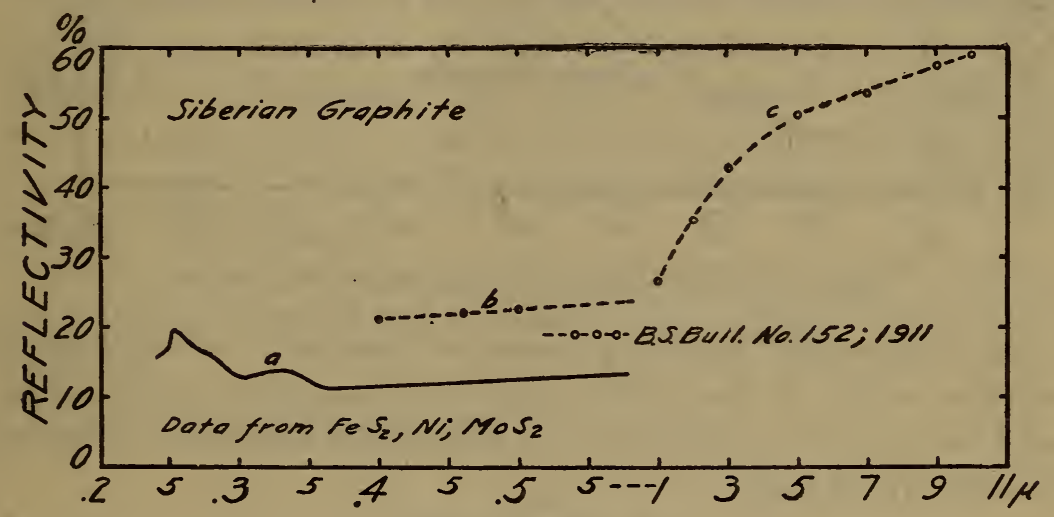

FIG. 5.-Spectral reflectivity of graphitized carbon-Siberian graphite

in curve $c$ of Figure 5. In the visible spectrum the reflectivity seems to vary uniformly, as shown in curve $b$, Figure 5 .

In the ultra-violet spectrum there appears to be a small reflectivity maximum in the region of $260 \mathrm{~m} \mu$, curve $a$, Figure 5 . For obtaining this curve the sample was freshly polished, but the surface was not so highly polished as is possible, and hence the absolute values are not so high as previously observed.

In the infra-red graphite seems to have the reflective properties of metals, while in the ultra-violet it seems to reflect selectively, as observed in the sulphides having a high metallic luster.

\section{DURALUMIN}

The sample examined was obtained from the Aluminum Co. of America, and while not sold under the above trade name it had the constituents of that alloy. 
The chemical analysis of this particular sample was as follows: 94.19 Al, 4.2 Cu, 0.37 Fe, 0.5 I Mn, 0.54 Mg, and o.19 Si (Zn, Sn, and $\mathrm{Ni}$ not detected).

The surface was not as highly polished as that of the magnalium mirror (A29) previously examined, ${ }^{16}$ and hence the absolute values are lower. However, the relative values of the spectral reflectivity are of the most importance. As shown in curve $a$ of Figure

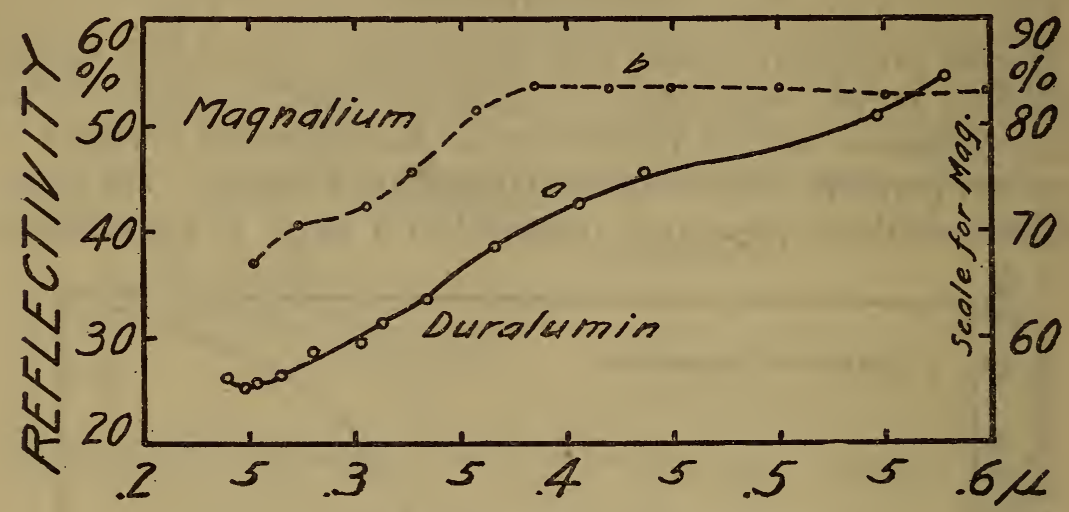

FIG. 6.-Spectral reflection of duralumin and magnalium

6 , the ultra-violet reflecting power of duralumin is much lower (relative to that of the visible spectrum) than that of magnalium, and hence is not so desirable as a magnalium mirror for reflecting the ultra-violet rays.

\section{SUMMARY}

Previous investigations of the ultra-violet reflective properties of materials were confined principally to metals and alloys. The present investigation was undertaken for the purpose of obtaining data on the ultra-violet reflecting power of the sulphides of metals having a high metallic luster.

The materials examined are the sulphides of lead, molybdenum, iron, and antimony in the form of the natural minerals, galena, molybdenite, pyrites, and stibnite.

The apparatus used in this investigation consisted of a quartz spectroradiometer and a vacuum thermopile. The source of radiation was a quartz mercury arc lamp. The measurements were extended to $240 \mathrm{~m} \mu$ in the ultra-violet.

In previous communications attention was called to what appears to be a common property of all metals, viz, a low reflecting

16 Waltenberg and Coblentz, B. S. Sci. Papers (No. ${ }^{6} 6_{3}$ ), 15, p. 653 ; 1920. 
power throughout the ultra-violet and the visible spectrum, followed by a high and uniform reflecting power in the infra-red.

In contrast with the metals, the results of the present investigation show that the sulphides of those metals which have a high metallic luster have their maximum reflectivity in the ultraviolet, followed by a uniform and, generally, a much lower reflectivity in the visible and in the infra-red spectrum.

The sulphides of antimony, iron, and lead (stibnite, pyrites, and galena) have a wide maximum of selective reflection in the spectral region of 310 to $325 \mathrm{~m} \mu$. Molybdenite has four maxima of selective reflection at $260,450,610$, and $690 \mathrm{~m} \mu$, respectively.

The reflecting power of duralumin is lower than that of magnalium, in the ultra-violet.

Graphitized carbon, at least in the form of the natural Siberian mineral, has a maximum of selective reflection at $260 \mathrm{~m} \mu$ in the ultra-violet. Throughout the visible spectrum the reflecting power is fairly uniform, while in the infra-red the reflecting power increases rapidly with the wave length, attaining a value of 60 per cent or higher at i $2 \mu$.

The paper calls attention to a newly observed deterioration of the surfaces of metals and sulphides when exposed to ultra-violet radiation.

WASHINGTON, June I3, I924. 


